\title{
A Regularized Model-Based Optimization Framework for Pan-Sharpening
}

\author{
Hussein A. Aly, Senior Member, IEEE, and Gaurav Sharma, Fellow, IEEE
}

\begin{abstract}
Pan-sharpening is a common postprocessing operation for captured multispectral satellite imagery, where the spatial resolution of images gathered in various spectral bands is enhanced by fusing them with a panchromatic image captured at a higher resolution. In this paper, pan-sharpening is formulated as the problem of jointly estimating the high-resolution (HR) multispectral images to minimize an objective function comprised of the sum of squared residual errors in physically motivated observation models of the low-resolution (LR) multispectral and the HR panchromatic images and a correlation-dependent regularization term. The objective function differs from and improves upon previously reported model-based optimization approaches to pan-sharpening in two major aspects: 1) a new regularization term is introduced and 2) a highpass filter, complementary to the lowpass filter for the LR spectral observations, is introduced for the residual error corresponding to the panchromatic observation model. To obtain pan-sharpened images, an iterative algorithm is developed to solve the proposed joint minimization. The proposed algorithm is compared with previously proposed methods both visually and using established quantitative measures of SNR, spectral angle mapper, relative dimensionless global error in synthesis, Q, and Q4 indices. Both the quantitative results and visual evaluation demonstrate that the proposed joint formulation provides superior results compared with pre-existing methods. A software implementation is provided.
\end{abstract}

Index Terms - Pan-sharpening, satellite imagery, image fusion, spectral imaging.

\section{INTRODUCTION}

$\mathbf{S}$ ATELLITE based multi and hyperspectral image capture systems use on-board imaging sensors that vary in spatial resolution. Typical sensor configurations capture one panchromatic image with high spatial resolution and multiple spectral images with low spatial resolution. The panchromatic sensor is sensitive over a wide wavelength range and therefore provides no spectral resolution whereas each spectral image sensor is responsive only over a relatively narrow wavelength band that it resolves spectrally. The spectral sensors are designed with lower spatial resolution, which allows a better signal to noise

Manuscript received July 14, 2013; revised February 10, 2014 and April 7, 2014; accepted April 7, 2014. Date of publication April 16, 2014; date of current version May 7, 2014. The associate editor coordinating the review of this manuscript and approving it for publication was Dr. Brendt Wohlberg.

H. A. Aly was with the Department of Electrical and Computer Engineering, University of Rochester, Rochester, NY 14627-0126 USA. He is now with the Ministry of Defense, Military Technical College, Cairo 11787, Egypt (e-mail: haly@ieee.org).

G. Sharma is with the Department of Electrical and Computer Engineering, Department of Biostatistics and Computational Biology, Department of Oncology, University of Rochester, Rochester, NY 14627-0126 USA (e-mail: g.sharma@ieee.org).

This paper has supplementary downloadable material available at http://ieeexplore.ieee.org., provided by the author. The material consists of two files. The total size of the files is 143 MB. Contact g.sharma@ieee.org for further questions about this work.

Color versions of one or more of the figures in this paper are available online at http://ieeexplore.ieee.org.

Digital Object Identifier 10.1109/TIP.2014.2316641 because of the associated larger physical area over which light is captured for each pixel in the image sensor. Before they are used in further analysis, LR spectral images are commonly post-processed, to obtain versions that match the higher resolution sampling of the panchromatic image [1]. This process, commonly referred to as pan-sharpening, merges together the low resolution and spectral information captured in the spectral channels with HR detail from the panchromatic image.

The goal of pan-sharpening is to estimate images that would be captured by an ideal system where the spectral sensors retain their existing spectral sensitivities but have a higher spatial resolution matching that of the panchromatic sensor [2]. Techniques for pan-sharpening have been extensively researched and reported in the literature. Reviews summarizing the different approaches and comparative benchmarking data for the prominent methods can be found in [3]-[9]. Component substitution (CS) and multi-resolution analysis (MRA) are two of the dominant frameworks for pansharpening. The CS framework is characterized by the use of per-pixel transformation of the spectral channels to generate the pan-sharpened images, which is attractive because of its simplicity. The LR images are spatially interpolated to match the panchromatic image size and transformed into a "color" representation where perceptual detail concentrates within a single channel. This channel is replaced with a HR image derived from the panchromatic image and the inverse of the transform is applied to obtain pan-sharpened images. One of the earliest techniques used an intensity-hue-saturation (IHS) representation for thee spectral channels with substitution of the intensity channel. Since then, alternatives that better preserve spectral accuracy and/or generalize to more than three channels have been proposed, including the Brovey transform, principal component replacement, and the GramSchmidt (GS) transform [10] and a generalized IHS (GIHS) method [11]. Data adaptive versions of the GIHS approach and of the Gram-Schmidt approach designated GIHSA and GSA, respectively, are among the most promising current techniques in the CS framework [12].

Different from the per-pixel channel transformations used in the CS methodology, MRA based pan-sharpening techniques utilize spatial transformations. The basic methodology operates as follows. For each spectral channel, first, via a grayscale transformation (e.g., histogram matching) of the panchromatic image, a HR image is generated with global statistics matched to those of the captured LR spectral image. Next, a spatial multi-resolution transformation is applied to the synthesized image and the low-frequency subband data in the multi-resolution decomposition is replaced with information derived from the captured LR spectral image. Finally, the 
inverse multi-resolution transform recreates a pan-sharpened image for the channel in consideration. Within the broad MRA framework a number of different pan-sharpening schemes have been developed. A large majority of these use multi-scale wavelet transforms [13], although other MRA decompositions such as curvelets have also been explored. Recent work on pan-sharpening has explored hybrid approaches that combine elements of the CS and MRA frameworks. Several of these techniques are reviewed in [13] and [9].

While a majority of pan-sharpening methods adopt a CS, MRA, or a hybrid CS+MRA approach, techniques independent of these frameworks have also been proposed. Among these, most relevant to our discussion are methods that adopt a model-based optimization (MBO) framework, where pansharpening is posed as an optimization problem minimizing a cost function based on a model of the imaging sensor or of the interrelation between the captured LR spectral and HR panchromatic images [14]-[19]. In this paper, we propose a new pan-sharpening method in the MBO framework. We pose the problem of estimating the HR multispectral images, jointly, as the minimization of an objective function formed as the sum of three terms. The first two terms form a combined squared residual error in physically motivated observation models of the LR multispectral and the HR panchromatic images. The third term represents a correlation dependent regularization. We develop an iterative algorithm to solve the minimization with modest computational complexity. Compared with prior MBO methods for pan-sharpening, the proposed approach presents novelty via an objective function that combines: a) a new regularization term that incorporates high-frequency detail from the panchromatic image into the estimated $H R$ spectral image in a correlation weighted fashion, reducing to constrained least-squares regularization in the absence of correlation, b) a squared error term corresponding to the panchromatic observation model that includes a spatial high pass filter that removes the influence of this term on low spatial frequencies where the observed multi-spectral images provide a better model, and c) explicit models for both spatial blurring and down-sampling the observation model for the $L R$ spectral image. The first two of these innovations are new and have not been previously utilized in MBO methods. The third has previously been used in some [14], [17], [19] MBO based pan-sharpening methods. Other MBO pan-sharpening methods [15], [16] have used a simplification in which versions of the LR images interpolated to the HR sampling grid are considered the observed LR images. We further highlight the attributes that distinguish our work from the prior MBO methods in Section VI after we present details of our method, using the context we establish in our presentation for better elaboration. Using approximate frequency-domain analysis we help develop intuition regarding the utility of, and trade-offs between, the three individual terms in our proposed objective function also demonstrate that the algorithm exhibits correct behavior under idealized conditions. Results benchmarking and comparing the proposed method against the leading existing alternatives demonstrate its advantage: it offers superior performance in both visual comparison and in numerical metrics used for assessment of quality.
Part of this work has previously been presented in preliminary form in [20]. The present manuscript improves and extends the work in [20] via: (a) the addition of a regularization term to the objective function that is essential in the presence of noise and model uncertainty, (b) a more complete presentation of the development and implementation, (c) approximate analysis of the algorithm in the frequency domain, and (d) expanded benchmarking of the performance of both the proposed algorithm and previously proposed alternatives, and (e) an enhanced and more intuitive notational convention for the presentation.

The rest of this manuscript is organized as follows. Section II introduces our joint formulation of the pansharpening problem as a minimization problem. Section III develops an iterative minimization approach for solving the minimization in a computationally tractable fashion. Approximate analysis of the proposed method is presented in Section IV to provide some insight into its operation. Experimental results obtained using the proposed algorithm are presented in Section V. A discussion of the differences between the proposed approach and prior MBO methods is presented in Section VI along with a comparison of the computational complexity of the different methods. Concluding remarks bring the paper to a close in Section VII.

\section{Proposed Formulation of PAN-Sharpening AS AN OPTIMIZATION PROBLEM}

We begin with a physical model for the multispectral imaging system. A spatio-spectral distribution $r(\chi, v ; \lambda)$ of light intensity is incident upon the sensor image planes used for capturing the panchromatic and the spectral images, ${ }^{1}$ where the pair $(\chi, v) \in \mathbb{R}^{2}$ represents an orthogonal coordinate system for the sensor image plane aligned with the sensor sampling grid and $\lambda$ denotes the wavelength of light. A panchromatic image is obtained, using a sensor sensitive to a wide wavelength range and having a sampling interval $X$ along each dimension, represented as a 2D orthogonal lattice $\Lambda \stackrel{\text { def }}{=}\left\{\left(k_{1} X, k_{2} X\right) \mid k_{1}, k_{2} \in \mathbb{Z}\right\}$. The captured panchromatic image is represented as $p[\mathbf{x}]=\bar{p}\left(k_{1} X, k_{2} X\right)+\eta_{0}[\mathbf{x}], \quad \mathbf{x}=$ $\left(k_{1} X, k_{2} X\right) \in \Lambda$, where

$$
\bar{p}(\chi, v)=\mathcal{H}_{0}^{\Lambda}(\chi, v) * \int r(\chi, v ; \lambda) \tau_{0}(\lambda) d \lambda,
$$

with $\mathcal{H}_{0}^{\Lambda}(\chi, v)$ and $\tau_{0}(\lambda)$ representing, respectively, the point spread function (PSF) and the spectral responsivity of the panchromatic imager, $*$ representing the convolution operation, and $\eta_{0}[\mathbf{x}]$ denoting the noise in the observations. Simultaneously, $K$ spectral images are also acquired via imagers sensitive to narrow ${ }^{2}$ spectral bands on the sparser orthogonal sampling lattice $\Gamma \stackrel{\text { def }}{=}\left\{\left(k_{1} q X, k_{2} q X\right) \mid k_{1}, k_{2} \in \mathbb{Z}\right\}$ having a spatial-sampling interval $q X$ along each dimension, where $q>1$ so that the spectral channels have a lower resolution than the panchromatic. These captured spectral images

\footnotetext{
${ }^{1}$ Throughout this paper, we assume that the captured images are spatially registered using suitable techniques.

${ }^{2}$ Relative, to the panchromatic channel.
} 
are represented by $c_{i}[\mathbf{x}]=\bar{c}_{i}\left(k_{1} q X, k_{2} q X\right)+\eta_{i}[\mathbf{x}], \quad \mathbf{x}=$ $\left(k_{1} q X, k_{2} q X\right) \in \Gamma$, for $i=1,2, \ldots, K$, where

$$
\bar{c}_{i}(\chi, v)=\mathcal{H}_{i}^{\Gamma}(\chi, v) * \int r(\chi, v ; \lambda) \tau_{i}(\lambda) d \lambda,
$$

with $\mathcal{H}_{i}^{\Gamma}(\chi, v)$ and $\tau_{i}(\lambda)$ representing, respectively, the point spread function (PSF) and the spectral responsivity of the $i^{\text {th }}$ spectral imager, and $\eta_{i}[\mathbf{x}]$ denoting the noise in the observations for the $i^{\text {th }}$ spectral channel. When the panchromatic image $p$ and the spectral images $\left\{c_{i}\right\}_{i=1}^{K}$ are acquired by the same satellite, $q$ is typically an integer factor and $\Gamma \subset \Lambda$, which is the situation we focus on in this paper. Generalizations to rational downsampling factors between $\Lambda$ and $\Gamma$ are straightforward.

The PSF $\mathcal{H}_{0}^{\Lambda}(\chi, v)$ is matched with the dense sampling lattice $\Lambda$ and the PSFs $\mathcal{H}_{i}^{\Gamma}(\chi, v), i=1,2, \ldots, K$ are matched with the sparse sampling lattice $\Gamma$. Spatial resolution is maximized without introducing aliasing in the sampled images when these PSFs correspond to ideal low pass filters with a cut-off frequency corresponding to the Nyquist frequency for the corresponding lattices. Due to practical manufacturing and cost constraints actual PSFs deviate from this ideal behavior. For typical deployed systems, the modulation transfer function (MTF), which corresponds to the magnitude of the Fourier transform of the PSF, exhibits both a falloff from the maximum within the desired passband as one approaches the Nyquist frequency and a residual nonzero response above the Nyquist frequency that contributes to a (small) amount of aliasing in the captured imagery. Note that unlike some other imaging scenarios, the noise level usually varies quite significantly across the different spectral bands because of the significant differences in the noise sources and in the native sensitivity of the underlying sensor used to capture the images. As a result, differences as high as 3-dB are not uncommon for the SNR for the different spectral bands [21].

Given the observed HR panchromatic image $p[\mathbf{x}], \mathbf{x} \in \Lambda$ and the LR spectral images $\left\{c_{i}[\mathbf{x}]\right\}_{i=1}^{K}, \mathbf{x} \in \Gamma$, our objective is to recover HR spectral images $f_{i}[\mathbf{x}] \stackrel{\text { def }}{=} f\left(k_{1} X, k_{2} X\right), \quad \mathbf{x}=$ $\left(k_{1} X, k_{2} X\right) \in \Lambda$ for $i=1,2, \ldots, K$, where

$$
f_{i}(\chi, v)=\mathcal{H}_{i}^{\Lambda}(\chi, v) * \int r(\chi, v ; \lambda) \tau_{i}(\lambda) d \lambda .
$$

where $\mathcal{H}_{i}^{\Lambda}(\chi, v)$ defines a suitable PSF for the $i^{\text {th }}$ spectral channel matched with the HR sampling lattice $\Lambda$. More than one reasonable choice exist for $\mathcal{H}_{i}^{\Lambda}(\chi, v)$. One reasonable choice, for instance, is: $\mathcal{H}_{i}^{\Lambda}(\chi, v)=\mathcal{H}_{i}^{\Gamma}(\chi / q, v / q)$ for $i=1,2, \ldots, K$, in which case the PSF for each desired HR spectral image is defined by scaling the PSF for the actually captured corresponding spectral image by a factor $(1 / q)$ along each spatial direction, so that the corresponding modulation transfer function, and effective bandwidth, are scaled by a factor $q$, as is desirable when the sampling density is increased by $q$ (along each dimension). An alternate reasonable choice is to set, $\mathcal{H}_{i}^{\Lambda}(\chi, v)=\mathcal{H}_{0}^{\Lambda}(\chi, v)$ for $i=1,2, \ldots, K$, in which case the PSF for all of the desired HR spectral images is chosen to match the PSF for the panchromatic channel, which is natively captured at $\mathrm{HR}$.

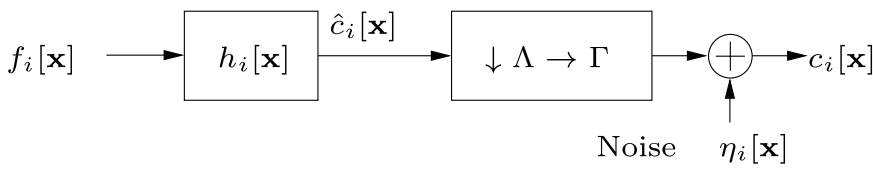

Fig. 1. Discrete domain observation model for the LR spectral image $c_{i}[\mathbf{x}]$ in terms of the corresponding $\mathrm{HR}$ image $f_{i}[\mathbf{x}]$.

The problem of estimating the HR spectral images $\left\{f_{i}[\mathbf{x}]\right\}_{i=1}^{K}, \quad \mathbf{x} \in \Lambda$, is a special version of the resolution enhancement/super-resolution problem [22]. Unlike typical single image super-resolution, however, for pan-sharpening, the panchromatic image $p[\mathbf{x}], \mathbf{x} \in \Lambda$ provides some of the high frequency spatial information that is missing in the captured LR spectral imagery. To proceed to formulate the pansharpening problem, we specify the observation model for the LR observed spectral images in the discrete domain as a lowpass filter $h_{i}[\mathbf{x}]$ on the lattice $\Lambda$ followed by downsampling to the lattice $\Gamma$, which is illustrated in Fig. 1. Using the standard stacked notation [23, p. 212], we obtain the operation in matrix-vector format as

$$
\mathbf{c}_{i}=\mathbf{H}_{i} \mathbf{f}_{i}+\boldsymbol{\eta}_{i},
$$

where $\mathbf{c}_{i}$ and $\mathbf{f}_{i}$ are the stacked notation vectors representing $c_{i}[\mathbf{x}]$ and $f_{i}[\mathbf{x}]$, respectively, and $\mathbf{H}_{i}$ is the rectangular matrix representing the low-pass filtering and sub-sampling, having essentially one row for every $q^{2}$ columns, ${ }^{3}$ and $\eta_{i}$ represents the noise in stacked format. If the filter $\mathcal{H}_{i}(\chi, v)$ is an ideal band-limited filter matched to the Nyquist bandwidth for the lattice $\Gamma$, the discrete domain observation model is exact [24]. Because ideal filters are non-realizable and due to other limitations, practical systems use non-ideal filters. The filters $h_{i}[\mathbf{x}], i=1,2, \ldots, K$ can then be optimally designed as in [25] using knowledge of $\mathcal{H}_{i}^{\Gamma}(\chi, v)$ provided as part of the system specifications. For our formulation, analogous to the filters $h_{i}[\mathbf{x}], i=1,2, \ldots, K$ for the multispectral channels, we also define a lowpass filter $h_{0}[\mathbf{x}]$ on $\Lambda$ for the panchromatic image such that $h_{0}[\mathbf{x}] * p[\mathbf{x}]$ downsampled to $\Gamma$, approximates capture of a LR panchromatic image on the lattice $\Gamma$ via a filter with impulse response $\mathcal{H}_{0}^{\Gamma}(\chi, v) \stackrel{\text { def }}{=} \mathcal{H}_{0}^{\Lambda}[q \chi, q v]$.

We now formulate pan-sharpening as the estimation of $\left\{f_{i}[\mathbf{x}]\right\}_{i=1}^{K}$ for $\mathbf{x} \in \Lambda$ by combining the LR and partly aliased information in $\left\{c_{i}[\mathbf{x}]\right\}_{i=1}^{K}, \quad \mathbf{x} \in \Gamma$ with the higher resolution spatial information available in the panchromatic image $p[\mathbf{x}], \mathbf{x} \in \Lambda$, exploiting, in the process, the spectral correlation between the panchromatic and the spectral channels due to their overlap. The specification of the spectral sensitivities of the panchromatic and the individual spectral channels provides a model for the spectral correlation. Specifically, we write

$$
\tau_{0}(\lambda)=\sum_{i=1}^{K} \omega_{i} \tau_{i}(\lambda)+\zeta(\lambda),
$$

where the summation represents the best attainable approximation to the spectral responsivity $\tau_{0}(\lambda)$ for the panchromatic

\footnotetext{
${ }^{3}$ Because our final implementations are all based on discrete filtering operations, we leave unspecified the sizes of the images and the corresponding vectors and matrices.
} 
channel in terms of the spectral responsivities $\left\{\tau_{i}(\lambda)\right\}_{i=1}^{K}$ for the $K$ spectral channels, treated as a basis, and $\zeta(\lambda)$ represents the residual error in the approximation. The weights $\left\{\omega_{i}\right\}_{i=1}^{K}$ can be obtained from the specification of the spectral sensitivities via least squares regression. If the specified PSFs $\mathcal{H}_{i}^{\Lambda}(\chi, v), \quad i=1,2, \ldots, K$ for the desired HR spectral images are identical to the PSF $\mathcal{H}_{0}^{\Lambda}(\chi, v)$ for the observed panchromatic image, one can readily see that the spectral relation in (5) induces a corresponding relation for the (noisefree) HR images

$$
\bar{p}[\mathbf{x}] \stackrel{\text { def }}{=} \bar{p}\left(k_{1} X, k_{2} X\right)=\sum_{i=1}^{K} \omega_{i} f_{i}[\mathbf{x}]+\zeta[\mathbf{x}],
$$

where $\mathbf{x}=\left(k_{1} X, k_{2} X\right)$, the image $\zeta[\mathbf{x}]$ is defined as the one corresponding to an imager with a virtual spectral sensitivity $\zeta(\lambda)$, PSF $\mathcal{H}_{0}^{\Lambda}(\chi, v)$, and sampling lattice $\Lambda$. For $i=1,2, \ldots, K$, we also denote by $\kappa_{i}$ the projection of the panchromatic channel spectral sensitivity onto the $i^{\text {th }}$ spectral channel sensitivity, specifically,

$$
\kappa_{i} \triangleq \frac{\left\langle\tau_{i}(\lambda), \tau_{0}(\lambda)\right\rangle}{\left\|\tau_{0}(\lambda)\right\|}=\frac{\int_{-\infty}^{\infty} \tau_{0}(\lambda) \tau_{i}(\lambda) d \lambda}{\sqrt{\int_{-\infty}^{\infty} \tau_{0}^{2}(\lambda) d \lambda}} .
$$

Next, on the lattice $\Lambda$, for $i=0,1,2, \ldots, K$, we define the complementary high-pass filter for the low pass filter $h_{i}[\mathbf{x}]$, by $g_{i}[\mathbf{x}]=\delta[\mathbf{x}]-h_{i}[\mathbf{x}]$, where $\delta[\cdot]$ represents the (Kronecker) delta function. Finally, we formulate pan-sharpening as the joint optimization:

$$
\begin{aligned}
\grave{\mathbf{f}}= & \arg \min _{\breve{\mathbf{f}}} \mathcal{J}(\breve{\mathbf{f}}, \mathbf{c}, \mathbf{p}), \\
\mathcal{J} \triangleq & \sum_{i=1}^{K}\left\|\mathbf{H}_{i} \mathbf{f}_{i}-\mathbf{c}_{i}\right\|^{2}+\alpha\left\|\mathbf{G}_{0}\left(\sum_{i=1}^{K} \omega_{i} \mathbf{f}_{i}-\mathbf{p}\right)\right\|^{2} \\
& +\sum_{i=1}^{K} \theta_{i}\left\|\mathbf{G}_{i}\left(\mathbf{f}_{i}-\kappa_{i} \mathbf{p}\right)\right\|^{2},
\end{aligned}
$$

where we use the stacked notation [23, p. 212] to compactly represent images as the corresponding vectors and the filtering (and downsampling) operations as matrices. We also, re-use the terms introduced in (4) and add the notation $\mathbf{p}$ to denote the panchromatic image $p[\mathbf{x}]$ in stacked form, the matrix $\mathbf{G}_{i}$ to denote filtering by $g_{i}[\mathbf{x}]$ for $i=0,1, \ldots, K$, and $\breve{\mathbf{f}}$ and $\mathbf{c}$ to jointly represent the complete set of HR and LR spectral images $\left\{\mathbf{f}_{i}\right\}_{i=1}^{K}$ and $\left\{\mathbf{c}_{i}\right\}_{i=1}^{K}$, respectively. The parameters $\theta_{1}, \theta_{2}, \ldots, \theta_{K}$ are scalar nonnegative regularization factors for which, suitable values can be determined by crossvalidation [26]. For our immediate discussion, we assume that the parameter $\alpha$ introduced in (8) is chosen to be $\alpha=1$; subsequently we introduce other values allow us to formulate an alternative pan-sharpening approach for benchmarking purposes. The individual terms in the objective function are described and motivated next.

The first summation term $\sum_{i=1}^{K}\left\|\mathbf{H}_{i} \mathbf{f}_{i}-\mathbf{c}_{i}\right\|^{2}$ in the objective function $\mathcal{J}$ represents the data-fidelity requirement for the $K$ observed spectral channels under the observation model of Fig 1. The second term $\left\|\mathbf{G}_{0}\left(\sum_{i=1}^{K} \omega_{i} \mathbf{f}_{i}-\mathbf{p}\right)\right\|^{2}$ (with $\alpha=1$ ) represents the requirement for consistency of the
$K$ estimated HR images with the spectral correlation model for the panchromatic image in (6), where this requirement is imposed only on the high pass filtered components of the images. The high pass filtering is beneficial because the residual $\zeta[\mathbf{x}]$ in (6) is ignored, it is helpful not to include in the second term lower frequency components that would erroneously compete with the more accurate low frequency spectral information included through the first term. This idea, specifically motivates the use of the complementary filter $g_{0}[\mathbf{x}]$ in the second term in objective function in (8), which is represented by the matrix $\mathbf{G}_{0}$. The third and final term $\sum_{i=1}^{K} \theta_{i}\left\|\mathbf{G}_{i}\left(\mathbf{f}_{i}-\kappa_{i} \mathbf{p}\right)\right\|^{2}$ in the objective function $\mathcal{J}$ in (8) represents a regularization term that is required because the pan-sharpening problem is ill-posed. Specifically, the high frequency components of the $K$ HR spectral images in $\breve{\mathbf{f}}$ are under-determined given the observed data because the second term of the objective function constrains only the sum of the $K$ highpass filtered HR spectral images. The regularization (third) term is carefully designed to provide spectral correlation dependent regularization. When $\kappa_{i}=0$, the regularization term reduces to $\left\|\mathbf{G}_{i} \mathbf{f}_{i}\right\|^{2}$ which is constrained least-squares regularization (independent of $\mathbf{p}$ ). For a nonzero value of $\kappa_{i}$ the regularization is guided by the correlation between $\mathbf{G}_{i} \mathbf{f}_{i}$ and $\mathbf{G}_{i} \mathbf{p}$ and incorporates spatial detail from the panchromatic channel into the spectral channel. A suitable value of the regularization factors $\theta_{1}, \theta_{2}, \ldots, \theta_{K}$ can be determined via cross-validation [26]; a small value is expected to be optimal to give precedence to the observed data terms. The spatial detail introduced in the pan-sharpened images via optimization of (8) is determined by the combination of the second and third terms. The third term introduces spatial detail but is constrained by the second term that ensures that high frequency spatial detail in the panchromatic image $p[\mathbf{x}]$ must be appropriately apportioned to the spectral channels and cannot be excessively re-utilized nor unduly ignored. With these three terms, the objective function in (8) combines the dual goals of spectral and spatial consistency with the observed data.

Note that by setting the parameter $\alpha=0$ we can eliminate the second term in our objective function in (8). With this setting, we can readily see that the optimization of (8) separates into $K$ individual optimizations and corresponds to a per-channel formulation of pan-sharpening that retains all other aspects of our proposed framework. This highlights the versatility of the proposed framework and also allows us to evaluate the benefit of the proposed joint formulation by comparing pan-sharpened images obtained for the joint $(\alpha=1)$ and the per-channel $(\alpha=0)$ scenarios. Other variants are also possible by varying the correlation terms $\kappa_{i}$ and the highpass filter $\mathbf{G}_{0}$ in the third term and second terms, respectively, of the objective function. These variants allow us to estimate the usefulness of the different components used in our objective function and will be explored for this purpose in Section V.

\section{ItERATIVE Minimization Algorithm}

The objective function in (8) is readily seen to be convex (in fact, quadratic) in the optimization variables in $\breve{\mathbf{f}}$. 


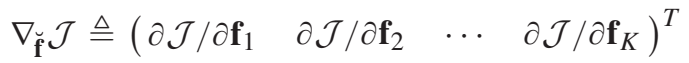

$$
\begin{aligned}
& =2\left(\begin{array}{c}
\mathbf{H}_{1}^{T}\left(\mathbf{H}_{1} \mathbf{f}_{1}-\mathbf{c}_{1}\right)+\alpha_{1} \mathbf{G}_{0}^{T} \mathbf{G}_{0}\left(\sum_{i=1}^{K} \omega_{i} \mathbf{f}_{i}-\mathbf{p}\right)+\theta_{1} \mathbf{G}_{1}^{T} \mathbf{G}_{1}\left(\mathbf{f}_{1}-\kappa_{1} \mathbf{p}\right) \\
\mathbf{H}_{2}^{T}\left(\mathbf{H}_{2} \mathbf{f}_{2}-\mathbf{c}_{2}\right)+\alpha_{2} \mathbf{G}_{0}^{T} \mathbf{G}_{0}\left(\sum_{i=1}^{K} \omega_{i} \mathbf{f}_{i}-\mathbf{p}\right)+\theta_{2} \mathbf{G}_{2}^{T} \mathbf{G}_{2}\left(\mathbf{f}_{2}-\kappa_{2} \mathbf{p}\right) \\
\vdots \\
\vdots \\
\mathbf{H}_{K}^{T}\left(\mathbf{H}_{K} \mathbf{f}_{K}-\mathbf{c}_{K}\right)+\alpha_{K} \mathbf{G}_{0}^{T} \mathbf{G}_{0}\left(\sum_{i=1}^{K} \omega_{i} \mathbf{f}_{i}-\mathbf{p}\right)+\theta_{K} \mathbf{G}_{K}^{T} \mathbf{G}_{K}\left(\mathbf{f}_{K}-\kappa_{K} \mathbf{p}\right)
\end{array}\right)
\end{aligned}
$$

The optimal estimate can therefore be obtained as the solution to the system of equations $\nabla_{\breve{f}} \mathcal{J}=0$, where the gradient $\nabla_{\breve{f}} \mathcal{J}$ of our objective function in (8) is obtained, via relatively straightforward algebra, as (9), shown at the top of this page, where we introduce $\alpha_{i} \triangleq \alpha \omega_{i}$ to allow for more compact representation of the equations. Although, these equations are linear, the number of variables in $\mathbf{f}$, i.e., the number of spectral channels times the number of pixels in each HR spectral image, and corresponding sizes of the matrices involved are too large to allow for a direct solution using our spatial domain matrix representation. ${ }^{4}$ Unlike the typical image restoration setting, a closed form solution can also not be obtained by transforming to the frequency domain because of the downsampling operation in the operators $\mathbf{H}_{i}$.

We therefore develop a gradient-descent [27] based iterative algorithm for optimization along with an efficient filter based implementation. From (9), it follows that the gradient descent iteration with a numerical step size $\Delta T$ can be written in perchannel (although still coupled) form as

$$
\begin{aligned}
& \mathbf{f}_{i}^{(n+1)} \\
& =\mathbf{f}_{i}^{(n)}-\Delta T\left(\mathbf{H}_{i}^{T}\left(\mathbf{H}_{i} \mathbf{f}_{i}^{(n)}-\mathbf{c}_{i}\right)+\alpha_{i} \mathbf{G}_{0}^{T} \mathbf{G}_{0}\left(\sum_{j=1}^{K} \omega_{j} \mathbf{f}_{j}^{(n)}-\mathbf{p}\right)\right. \\
& \\
& \left.+\theta_{i} \mathbf{G}_{i}^{T} \mathbf{G}_{i}\left(\mathbf{f}_{i}^{(n)}-\kappa_{i} \mathbf{p}\right)\right),
\end{aligned}
$$

where ${ }^{T}$ denotes matrix transpose, and the superscript $\cdot(n)$ denotes the iteration index.

The sizes or the matrices and vectors involved do not allow for a literal implementation of the iteration in (10). Instead, a memory and computation efficient implementation is obtained by realizing the required iterations via filtering and down/up sampling stages. The filtering equivalents corresponding to $\mathbf{G}_{i}$ and the filtering and downsampling interpretation for $\mathbf{H}_{i}$ have already been discussed. The operation $\mathbf{G}_{i}^{T}$ is a convolution matrix corresponding to the space-reversed PSF $g_{i}[-\mathbf{x}]$. If $g_{i}$ is quad-symmetric $\left(g_{i}[\mathbf{x}]=g_{i}[-\mathbf{x}]\right)$, then $\mathbf{G}_{i}^{T}=\mathbf{G}_{i}$. The matrix $\mathbf{H}_{i}^{T}$ represents up-sampling from $\Gamma$ to $\Lambda$ followed by convolution with the space reversal filter $h_{i}[-\mathbf{x}]$. The block diagram for the practical implementation of the update step in (10) for the $i^{\text {th }}$ spectral channel image is shown in Fig. 2. The algorithm is initialized by setting $\mathbf{f}_{i}^{(0)}$ to the

\footnotetext{
${ }^{4}$ For imagery from the IKONOS satellite imaging system, a typical set of four spectral images, represented at the panchromatic resolution at typically captured image sizes, has over 400 million pixels in aggregate.
}

image obtained by interpolation of $\mathbf{c}_{i}$ for $i=1,2 \ldots, N$, specifically bicubic interpolation in our implementation.

Because the objective function in (8) is convex, the iterations defined by (10) are guaranteed to converge to the (global) minimum provided the step size $\Delta T$ is chosen suitably. Specifically, a line search procedure [27] for the step size $\Delta T$ ensures monotonic convergence in our problem setting. We can also adopt the standard convergence criterion used in iterative optimization to terminate the iterations when both the change in images from one iteration to the next and the improvement in the objective function are smaller than pre-determined thresholds. In practice, however, these measures are unnecessary because the bicubic interpolation provides a good initial starting point. In our implementation, we therefore eliminate the per-iteration computational cost for estimating step size and testing for convergence by using a constant iteration count $N_{\max }$ and a fixed schedule for the step size $\Delta T$. Specifically, we use a fixed step size $\Delta T=\Delta T_{0}$ for an initially determined number $N_{0}$ of iterations and then geometrically scale the step size for iterations $N_{0}+1$ through $N_{\max }$, i.e., use a step size $\Delta T=\gamma^{n-N_{0}} \Delta T_{0}$ for iteration $n$ when $n>N_{0}$. The values $\Delta T_{0}, N_{\max }, N_{0}$, and $\gamma$ are empirically determined as described in Section $\mathrm{V}$ and in the Supplementary data (Section S.VI).

\section{Approximate Frequency Domain Analysis}

The behavior of the proposed algorithm can be approximately analyzed by considering the solution for the system $\nabla_{\breve{f}} \mathcal{J}=0$ in the frequency domain using the expression for the gradient in (9). Specifically, denoting by $[u, v]$ the two orthogonal frequency variables associated with the two spatial dimensions in $\mathbf{x}$, we can consider the discrete-space frequency region $[0,0.5] \times[0,0.5]$ associated with the fine lattice $\Lambda$, where a discrete frequency values of 0 and 0.5 correspond to continuous space frequency values of 0 and $1 /(2 X)$, respectively, and intermediate frequency values scale linearly to cover this range. To understand the behavior of the proposed algorithm, we note that the low pass filters $h_{i}[\mathbf{x}]$ have a unity response in the low frequency domain $[0,1 /(2 q)-B] \times$ $[0,1 /(2 q)-B]$ and near zero response in the high frequency region $[1 /(2 q)+B, 0.5] \times[1 /(2 q)+B, 0.5]$ where $2 B$ represents the transition band for these filters. The complementary high-pass filters $g_{i}[\mathbf{x}]$ exhibit the opposite behavior.

In the low-frequency region, i.e., $[u, v] \in[0,1 /(2 q)-B] \times$ $[0,1 /(2 q)-B]$, the second and third terms in the summations representing the gradients in (9) are zero because $G_{i}[u, v] \approx 0$ in these regions for all $i=0,1, \ldots, K$. In this region of 


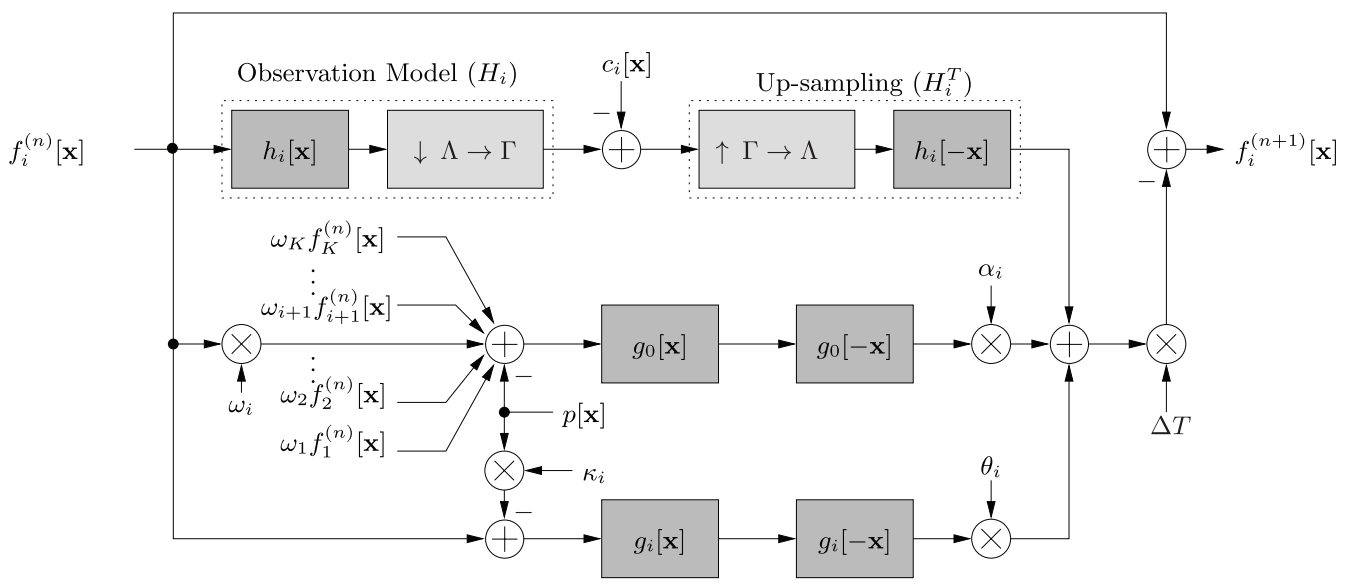

Fig. 2. Practical implementation of (10) for updating the $i^{\text {th }}$ spectral channel image in an iteration of the proposed pan-sharpening method.

frequency space, therefore the solution is determined entirely by the first data term. Using the fact that $H_{i}[u, v] \approx 1$ in these regions of frequency space, we can see that

$$
\grave{F}_{i}[u, v] \approx c_{i}[q u, q v] .
$$

Thus, in this low-frequency region, the estimated HR spectral images are completely consistent with the observed LR spectral images, as is desirable.

In the high-frequency region, i.e., $[u, v] \in[1 /(2 q)+$ $B, 0.5] \times[1 /(2 q)+B, 0.5]$, the first term in the summations representing the gradients in (9) are zero because $H_{i}[u, v] \approx 0$ in these regions for all $i=0,1, \ldots, K$. In this region of frequency space, therefore the solution is determined entirely by the second and third terms. Using the fact that $G_{i}[u, v]=1$ in these regions of frequency space for all $i=1,2, \ldots, K$, we can see that the estimated images satisfy the system of equations

$\alpha_{i}\left(\sum_{j=1}^{K} \omega_{j} \grave{F}_{j}[u, v]-P[u, v]\right)+\theta_{i}\left(\grave{F}_{i}[u, v]-\kappa_{i} P[u, v]\right) \approx 0$,

for $i=1,2, \ldots, K$.

For the per-channel formulation for pan-sharpening in our framework $(\alpha=0)$, (12) reduces to $\grave{F}_{i}[u, v]=\kappa_{i} P[u, v]$, i.e., the high frequency components of individual channels are determined purely by the correlations between panchromatic image and the individual spectral images. On the other hand, for our proposed joint formulation $(\alpha=1)$ under the typical situation where $\theta_{i}<<1, \forall i$, we see that resulting solution obtains the high-frequency components of the spectral images based on the correlations between the panchromatic image and the individual spectral images, while strongly enforcing consistency between the spectral and panchromatic components implied by (5). It is instructive to consider a few special cases for the joint scenario $(\alpha=1)$ :

- When $\omega_{i}=0$, i.e., the $i^{\text {th }}$ channel makes no contribution to the panchromatic image, the first term drops out and we have $\grave{F}_{i}[u, v]=\kappa_{i} P[u, v]$, the image is determined purely by the correlation between the spectral and the panchromatic channels.
- If $\kappa_{i}=0$ then (it can be readily seen that) we also have $\omega_{i}=0$ and in this case we have $\grave{F}_{i}[u, v]=0$, i.e., a smooth estimate is favored when no correlated data is available for the high frequencies.

- When the sensitivities corresponding to the $K$ spectral images are orthogonal (i.e., non-overlapping) and the panchromatic channel sensitivity $\tau_{0}(\lambda)$ is a weighted sum of some selection of these, say,

$$
\tau_{0}(\lambda)=\sum_{j \in \mathcal{S}} w_{j} \tau_{j}(\lambda)
$$

Then the solution to (12) reduces to

$$
\grave{F}_{i}[u, v]= \begin{cases}0 & j \notin \mathcal{S} \\ \sqrt{\frac{\left(w_{i}\left\|\tau_{i}(\lambda)\right\|\right)^{2}}{\sum_{j \in S}\left(w_{j}\left\|\tau_{j}(\lambda)\right\|\right)^{2}}}\left\|\tau_{i}(\lambda)\right\| P[u, v] & j \in \mathcal{S} .\end{cases}
$$

That is, the energy in $P[u, v]$ is allocated in an "energy proportional" fashion to the spectral bands that form the panchromatic band and other spectral bands have their high frequency components set to zero.

- A special case of the above arises when the $K$ spectral bands are unit-energy equi-bandwidth splits of a spectrally flat panchromatic channel, we have $\omega_{i}=1$ and $\kappa_{i}=1 / \sqrt{K}$ for $i=1,2, \ldots, K$. The solution to (12) then reduces to $\grave{F}_{i}[u, v]=(1 / \sqrt{K}) P[u, v]$, i.e., the "detail information" contained in the high frequency components in $p[\mathbf{x}]$ is apportioned equally in energy among the estimated spectral channels.

In the transition region where the one of the orthogonal spatial frequency components lies in $[1 /(2 q)-B, 1 /(2 q)+B]$, all three of the terms contribute to the estimates and we cannot readily obtain an expression for the estimates but from continuity arguments can see that the solution tends to the low and high frequency estimates at the appropriate boundaries and intermediate behavior can be expected in between.

We note that our analysis excludes consideration of any aliasing in the process of downsampling from $\Lambda$ to $\Gamma$. If the discrete filters $h_{i}[\mathbf{x}]$ are designed based on known specifications of the continuous time spatial filters $\mathcal{H}_{i}^{\Gamma}(\chi, v)$ 
and $\mathcal{H}_{i}^{\Lambda}(\chi, v)$, the aliasing can partly be comprehended and exploited in the pan-sharpening process as has been demonstrated for the related upsampling problem in [28].

\section{RESULTS}

To evaluate our proposed pan-sharpening method, we use imagery from the IKONOS multispectral imaging satellite [29], for which details of the system specifications, including the spectral responsivities and point-spread function characteristics are publicly available [21] along with a number of image datasets [30] comprised of coarsely registered pan and multispectral images at their native capture resolutions. Together these data provide an ideal test set for evaluating our proposed algorithm and for benchmarking its performance against previously proposed pan-sharpening alternatives. The IKONOS satellite has five imaging channels: a panchromatic channel $p[\mathbf{x}]$ with a nominal ground resolution of $X=1 \mathrm{~m}$ along each dimension and spanning the spectral range ${ }^{5}$ from 525.8 through $928.5 \mathrm{~nm}$ and $K=4$ multispectral bands with a nominal ground resolution of $4 X=4 m$ along each dimension (i.e., $q=4$ ) and having spectral bandwidths for the channels as follows [21]: (a) MS-1 (Blue) $c_{1}[\mathbf{x}]$, 444.7-516.0 nm, (b) MS-2 (Green) $c_{2}[\mathbf{x}], 506.4-595.0 \mathrm{~nm}$, (c) MS-3 (Red) $c_{3}[\mathbf{x}], 631.9-697.7 \mathrm{~nm}$, and (d) MS-4 (VNIR) $c_{4}[\mathbf{x}], 757.3-852.7 \mathrm{~nm}$. Plots of the normalized spectral sensitivities are included in the Supplementary data (Fig. S.1). For processing and computation, the 11 bit pixel data was converted to a floating point values by a linear mapping with the digital value of 2047 represented as 1.0. Output images were linearly mapped to an 8 bit 0 to 255 scale to facilitate viewing and comparison on common 8 bit display systems. For computing numerical benchmarks for comparing different methods, required "ground truth" was generated in the usual manner [1], [16], [31] by lowpass filtering and downsampling the panchromatic and the spectral data by a factor of 4 along each dimension using the system MTF parameters. The spectral images generated from this procedure are then used as the LR spectral observations and the original captured spectral images serve as HR ground truth data. Accordingly, the simulations use $\mathcal{H}_{i}^{\Lambda}(\chi, v)=\mathcal{H}_{i}^{\Gamma}(\chi / q, v / q)$ for $i=1,2, \ldots, 4$ for our spectral channels. For our algorithmic implementation (shown in Fig. 2), we realize the filters $h_{i}[\mathbf{x}], i=1,2, \ldots, 4$ as zero-phase finite-impulse-response (FIR) designed according to the specifications for the IKONOS system [21] via the optimization methodology proposed in [25]. Additional detail, including frequency responses for these filters, is provided in the Supplementary data accompanying this paper (Table S.I and Fig. S.2).

Required parameters for our pan-sharpening algorithm are obtained as follows. Using the publicly available specification data for the spectral sensitivities with least squares regression, we obtain the weights ${ }^{6} \omega_{1}=0.04, \omega_{2}=0.18, \omega_{3}=0.21$, and $\omega_{4}=0.34$. From the same data, we have $\kappa_{1}=0.039$,

\footnotetext{
${ }^{5}$ Stated spectral bandwidths correspond to full-width at half-max.

${ }^{6}$ Although these weights are obtained purely from the spectral responsivity data for the sensors, they are close in values to those obtained in [12] by regression over a set of sample images.
}

$\kappa_{2}=0.091, \kappa_{3}=0.092$, and $\kappa_{4}=0.152$. Through empirical observation of the convergence behavior of the algorithm, the parameter values for the gradient descent iterations were set to an initial step size of $\Delta T_{0}=4$, a maximum iteration count of $N_{\max }=50$, with a geometric reduction in the step size by a factor $\gamma=0.95$ for iterations greater than $N_{0}=20$. With these parameters, the iterations exhibited reasonably fast and almost monotone convergence, as is illustrated in in the Supplementary data (Section S.VI).

We compare the performance of the proposed (Prop) algorithm against: (a) bicubic interpolation (BC), (b) prominent pan-sharpening approaches in the CS and MRA frameworks, and (c) a recently proposed alternative MBO technique for pan-sharpening. Specifically, the benchmarked methods include: generalized IHS (GIHS) [11], Gram-Schmidt ${ }^{7}$ (GS) [10], multiscale wavelets (MSW) [2], with 3 levels ${ }^{8}$ for the wavelet decomposition (MSW3), GIHS adaptive (GIHSA) [12], GS adaptive (GSA) [12], and a recently reported alternative $\mathrm{MBO}$ approach that uses a total variation regularizer (MBTV) [19]. We note that [19] also includes a number of comparisons of the MBTV approach against other MBO methods and therefore also allows indirect comparison of our proposed approach against several other MBO methods. To highlight the contribution of the individual elements introduced in our framework, we also include four additional variants in our proposed framework as follows: Prop-PC-the per-channel variant obtained by setting $\alpha=0$; Prop-AP-the variant in which the second (joint) term in the objective function is not subject to the highpass filtering, i.e., $\mathbf{G}_{0}$ is set to the all pass identity operator $\mathbf{I}$ in the second term; Prop-CLS-the variant with $\kappa_{i}=0$ in which the regularization reduces to a constrained least squares (CLS) regularizer instead of the correlation dependent regularization; and Prop-NR-the variant with no regularization [20] obtained by setting $\theta_{1}=\theta_{2}=\theta_{3}=\theta_{4}=0$.

Because there is currently no single consensus metric for the evaluation of pan-sharpening [32], we consider multiple metrics for the assessment of pan-sharpened images. Specifically, for each of the pan-sharpening methods, we evaluate the fidelity of the estimated HR spectral images $\left\{\hat{f}_{i}[\mathbf{x}]\right\}_{i=1}^{K}$ to the original images $\left\{f_{i}[\mathbf{x}]\right\}_{i=1}^{K}$ by computing the following metrics:

- Per-spectral channel SNR defined (in dB) as [33, p. 129]

$$
S N R_{i}=10 \log _{10}\left(\frac{\sum_{\mathbf{x}}\left(f_{i}[\mathbf{x}]-\bar{f}_{i}\right)^{2}}{\sum_{\mathbf{x}}\left(d_{i}[\mathbf{x}]-\bar{d}_{i}\right)^{2}}\right)
$$

where $d_{i}[\mathbf{x}] \triangleq f_{i}[\mathbf{x}]-\hat{f}_{i}[\mathbf{x}]$ represents the difference (image) between the original and the estimated images and $\bar{f}_{i}\left(\bar{d}_{i}\right)$ denotes the spatial average of $f_{i}[\mathbf{x}]\left(d_{i}[\mathbf{x}]\right)$.

\footnotetext{
${ }^{7}$ We use the second GS variant described in [10], for which, the lowresolution panchromatic image used for computing the Gram-Schmidt transformation matrix is obtained by spatially degrading the high resolution panchromatic image based on the ratio between the resolutions. The first GS variant, which typically performs worse than second, uses a panchromatic image formed as a linear combination of the spectral images, with weights determined by regression.

${ }^{8}$ The multiscale wavelet technique was also tested with 4 levels of decomposition but performed worse than the reported 3 level case.
} 
- The spectral angle mapper distortion (SAM) [31] defined as the spatial average of the absolute angular difference $\Delta \Phi[\mathbf{x}]$ between the $K$-vectors $\mathbf{v}[\mathbf{x}]=\left[f_{1}[\mathbf{x}]\right.$, $\left.f_{2}[\mathbf{x}], \ldots, f_{K}[\mathbf{x}]\right]^{T}$ and $\hat{\mathbf{v}}[\mathbf{x}]=\left[\hat{f}_{1}[\mathbf{x}], \hat{f}_{2}[\mathbf{x}], \ldots, \hat{f}_{K}[\mathbf{x}]\right]^{T}$ corresponding to the true and the estimated values for the pixel $\mathbf{x}$, where $\Delta \Phi[\mathbf{x}]$ is computed as

$$
\Delta \Phi[\mathbf{x}] \triangleq \arccos \left(\frac{\mathbf{v}^{T}[\mathbf{x}] \hat{\mathbf{v}}[\mathbf{x}]}{\sqrt{\mathbf{v}[\mathbf{x}]^{T} \mathbf{v}[\mathbf{x}]} \sqrt{\hat{\mathbf{v}}^{T}[\mathbf{x}] \hat{\mathbf{v}}[\mathbf{x}]}}\right) .
$$

- The relative dimensionless global error in synthesis (ERGAS) [1], [34], [35], which can be expressed as

$$
\text { ERGAS } \triangleq 100 \frac{1}{q} \sqrt{\frac{1}{K} \sum_{i=1}^{K}\left(\frac{\sum_{\mathbf{x}}\left(f_{i}[\mathbf{x}]-\hat{f}_{i}[\mathbf{x}]\right)^{2}}{\left(\sum_{\mathbf{x}} f_{i}[\mathbf{x}]\right)^{2}}\right)} .
$$

- The average $Q^{\text {avg }}$ of the per channel universal image quality index [36], defined for the $i^{\text {th }}$ channel as [36]

$$
Q^{i} \triangleq \frac{4 \sigma_{f_{i} \hat{f}_{i}} \mu\left(f_{i}\right) \mu\left(\hat{f}_{i}\right)}{\left(\sigma_{f_{i}}^{2}+\sigma_{\hat{f}_{i}}^{2}\right)\left(\mu^{2}\left(f_{i}\right)+\mu^{2}\left(\hat{f}_{i}\right)\right)},
$$

where $\sigma_{a b}$ denotes the covariance between images $a$ and $b, \sigma_{a}^{2}$ the variance of image $a$, and $\mu(a)$ the mean of image $a$, each of the terms being estimated over a sliding window of size $W \times W$, which we indicated by a subscript as $Q_{W}^{\text {avg }}$ for our metric.

- The extension $Q_{W}^{4}$ of the universal image quality index (UQUI) [37] that aims to estimate jointly the quality of four band imagery using a quaternion representation to jointly represent the 4 spectral bands, where $W$ indicates the window size, as before.

The SNR and individual channel $Q^{i}$ measures are extensively used in the signal and image processing communities as mean-squared-error based and visual measures of quality, respectively. The SAM and ERGAS measures are commonly utilized in the remote sensing community. The SAM measure is motivated by the need to maintain the relative magnitudes of the spectral bands, which is important for identifying material characteristics, and ERGAS is considered as a global measure of the quality of the pan-sharpened image set with values below 3 being commonly considered acceptable [1]. The joint $Q_{W}^{4}$ measure comprehends correlation between the four channels while still allowing for a visually meaningful measure and has therefore also been adopted in the remote sensing community [37]. Larger values indicate better performance ( $\uparrow$ ) for SNR, $Q_{W}^{\mathrm{avg}}$, and $Q_{W}^{4}$ with the maximum value of 1 representing the ideal performance for $Q_{W}^{\mathrm{avg}}$, and $Q_{W}^{4}$. Smaller values indicate better performance $(\downarrow)$ for SAM and ERGAS, with 0 being the ideal value.

Table I compares the quantitative performance of the different algorithms using the different quality measures, where the best score for each measure is shown in bold. Results are shown individually for the four image sets available in the GeoEye dataset [30]. Because the measures vary significantly over the different images, we do not present average performance over the images. From the numerical measures we see that the proposed technique (Prop) outperforms the other techniques, offering either the best or close to the best performance with respect to almost all of the measures and for each of the image sets. The variant Prop-CLS in the proposed framework that uses a constrained least squares regularizer instead of the correlation dependent regularizer, offers the next best performance. Among the methods that are not variants of the proposed framework, GS, GSA, and GIHSA perform very close to each other and rank next after Prop-CLS. The results also highlight that the different components introduced in our framework are essential. The Prop-PC, Prop-NR, Prop-AP, and Prop-CLS variants that drop, respectively, the second "joint" data term, the third regularization term, the high pass filtering for the second term, and the correlation dependence for the regularization, each perform worse than the full proposed scheme (Prop). In particular, Prop-PC and Prop-AP exhibit severe degradation in performance compared with Prop whereas Prop-CLS suffers only a small degradation in performance and the non-regularized Prop-NR scheme performs well over the first couple of datasets but does quite poorly on the third and fourth datasets. The MBTV approach which is also an MBO method but uses a total variation regularizer, offers performance that is better than several of the alternative methods but not competitive with the proposed technique. Reasons for this have already elaborated in the context of the variants of the proposed method: specifically the two key innovations introduced in the objective function for the proposed method are absent in the MBTV approach.

Next we present images that allow visual evaluation and assessment of the proposed algorithm against the other methods benchmarked in Table I. In our comparisons, we also include images on the fine lattice $\Lambda$ obtained via bicubic interpolation (BC), in order to represent the baseline upon which pan-sharpening seeks to improve, and the panchromatic image (PAN) used in the pan-sharpening process. To allow detail in the images to be seen, we show a small corresponding region of the images obtained by each of the alternative techniques. The images corresponding to the R, G, B bands are combined as a single three-channel color image to allow compact presentation and also easy visualization of changes in relative magnitudes of the R, G and B channels, which are manifested as color shifts in the composite color images. Sample results for one dataset are shown in Fig. 3 for the pan-sharpening performed with the actual (non-simulated) recorded dataset. Fig. 3 includes the bicubic interpolated (BC), the original panchromatic image (PAN), and pan-sharpened images for the proposed method (Prop), for the CS methods GS, GIHSA, and for the MBTV MBO method. Results for additional pan-sharpening methods, for the NIR channel, for other datasets, and for the simulation scenario are presented separately in the Supplementary data accompanying this paper. For all these cases, the regularization parameters were set to $\theta_{1}=0.05, \theta_{2}=0.1, \theta_{3}=0.1, \theta_{4}=0.16$ to adapt to the varying SNR seen across channels. IMPORTANT: The images are best viewed in their native TIFF format versions submitted as supplementary material with the paper, where 
TABLE I

Quantitative Performance Measures of Different Pan-Sharpening Methods for the IKOnOS Sample Data Sets

"China-Sichuan $x x x x x \_0000000.2000 x x x x$ " Obtained From GeoEye [30]. For the Proposed Method

the Regularization Parameters Were Set to $\theta_{1}=0.04, \theta_{2}=0.1, \theta_{3}=0.15, \theta_{4}=0.04$ Based on

Cross-Validation [26]. The Metrics Are Computed Over the Irregular

Shaped SuPPort For VALid Data In THE IMAGe FiLes

\begin{tabular}{|c|c|c|c|c|c|c|c|c|}
\hline \multirow[t]{2}{*}{ Algorithm } & \multirow{2}{*}{$\begin{array}{l}\text { SAM } \\
\text { (deg) }\end{array}$} & \multirow[t]{2}{*}{ ERGAS } & \multicolumn{4}{|c|}{ SNR (dB) } & \multirow[t]{2}{*}{$Q_{32}^{\text {avg }}$} & \multirow{2}{*}{$Q_{32}^{4}$} \\
\hline & & & $\mathrm{R}$ & $\mathrm{G}$ & B & NIR & & \\
\hline \multicolumn{9}{|c|}{ Dataset: China-Sichuan 58204_0000000.20001116: $3,153,692$ valid pixels } \\
\hline $\mathrm{BC}$ & 3.6 & 2.70 & 24.95 & 26.75 & 29.31 & 17.56 & 0.693 & 0.693 \\
\hline GIHS & 4.2 & 3.06 & 22.64 & 22.94 & 22.72 & 18.52 & 0.727 & 0.838 \\
\hline GIHSA & 3.7 & 2.37 & 26.27 & 27.42 & 27.51 & 19.02 & 0.768 & 0.821 \\
\hline MSW3 & 4.2 & 2.58 & 25.09 & 25.78 & 25.18 & 18.85 & 0.710 & 0.804 \\
\hline GS & 3.8 & 2.39 & 25.75 & 27.04 & 27.82 & 19.05 & 0.749 & 0.813 \\
\hline GSA & 3.8 & 2.47 & 25.11 & 26.22 & 26.89 & 19.11 & 0.748 & 0.827 \\
\hline MBTV & 4.0 & 2.66 & 25.46 & 27.18 & 28.10 & 18.41 & 0.767 & 0.787 \\
\hline Prop & $\overline{3.0}$ & 2.14 & 26.67 & 28.83 & \begin{tabular}{|c|}
31.13 \\
\end{tabular} & 19.67 & 0.828 & 0.855 \\
\hline Prop-PC & 3.4 & 2.49 & 25.43 & 27.45 & 30.22 & 18.32 & 0.749 & 0.751 \\
\hline Prop-AP & 5.7 & 6.53 & 17.47 & 20.56 & 29.02 & 13.65 & 0.812 & 0.845 \\
\hline Prop-CLS & 3.0 & 2.16 & 26.45 & 28.67 & 31.04 & 19.64 & 0.823 & 0.854 \\
\hline Prop-NR & 3.3 & 2.28 & 25.11 & 27.24 & 28.32 & 19.89 & 0.764 & 0.848 \\
\hline \multicolumn{9}{|c|}{ Dataset: China-Sichuan 58205_0000000.20001003: $2,593,511$ valid pixels } \\
\hline $\mathrm{BC}$ & 3.2 & 2.47 & 20.62 & 22.39 & 26.37 & 14.93 & 0.621 & 0.597 \\
\hline GIHS & 3.6 & 2.89 & 18.38 & 19.05 & 18.70 & 16.47 & 0.701 & 0.833 \\
\hline GIHSA & 3.3 & 2.10 & 21.62 & 23.20 & 22.88 & 17.52 & 0.736 & 0.810 \\
\hline MSW3 & 3.8 & 2.46 & 19.75 & 20.91 & 19.90 & 17.76 & 0.660 & 0.815 \\
\hline GS & 3.9 & 2.43 & 19.37 & 21.44 & 21.96 & 17.07 & 0.674 & 0.788 \\
\hline GSA & 4.0 & 2.61 & 18.40 & 20.41 & 20.85 & 17.17 & 0.665 & 0.806 \\
\hline MBTV & 3.7 & 2.42 & 20.03 & 22.33 & 22.21 & 16.23 & 0.679 & 0.733 \\
\hline Prop & 2.5 & 1.77 & 22.23 & 24.57 & 27.79 & 18.88 & 0.819 & 0.862 \\
\hline Prop-PC & 3.1 & 2.26 & 21.30 & 23.29 & 27.52 & 15.67 & 0.705 & 0.677 \\
\hline Prop-AP & 4.3 & 5.58 & 17.20 & 20.58 & 26.99 & 17.26 & 0.799 & 0.853 \\
\hline Prop-CLS & 2.4 & 1.77 & 22.20 & 24.55 & 27.95 & 18.90 & 0.818 & 0.862 \\
\hline Prop-NR & 3.8 & 2.66 & 17.33 & 20.50 & 20.59 & 18.34 & 0.662 & 0.841 \\
\hline \multicolumn{9}{|c|}{ Dataset: China-Sichuan 58207_0000000.20000831: $4,598,200$ valid pixels } \\
\hline $\mathrm{BC}$ & 2.7 & 2.53 & 18.89 & 21.66 & 26.48 & 16.62 & 0.574 & 0.571 \\
\hline GIHS & 2.7 & 2.27 & 19.61 & 21.86 & 22.04 & 19.36 & 0.742 & 0.825 \\
\hline GIHSA & 2.6 & 2.08 & 20.28 & 23.07 & 23.55 & 19.63 & 0.755 & 0.811 \\
\hline MSW3 & 2.9 & 2.37 & 18.98 & 21.23 & 20.64 & 20.00 & 0.689 & 0.822 \\
\hline GS & 2.2 & 1.97 & 20.18 & 23.30 & 27.15 & 19.85 & 0.790 & 0.814 \\
\hline GSA & 2.2 & 1.97 & 20.13 & 23.26 & 27.05 & 19.90 & 0.803 & 0.829 \\
\hline MBTV & 3.5 & 2.80 & 17.59 & 21.14 & 20.86 & 17.96 & 0.652 & 0.723 \\
\hline Prop & 2.1 & 1.87 & 20.29 & 23.67 & 27.34 & 20.78 & 0.821 & 0.860 \\
\hline Prop-PC & 2.6 & 2.32 & 19.58 & 22.56 & 27.42 & 17.38 & 0.690 & 0.660 \\
\hline Prop-AP & 4.1 & 6.58 & 17.55 & 21.66 & 27.01 & 20.19 & 0.792 & 0.852 \\
\hline Prop-CLS & 2.0 & 1.85 & 20.41 & 23.77 & 27.62 & 20.80 & 0.824 & 0.860 \\
\hline Prop-NR & 3.9 & 3.37 & 13.94 & 18.33 & 18.70 & 20.20 & 0.602 & 0.833 \\
\hline \multicolumn{9}{|c|}{ Dataset: China-Sichuan 58208_0000000.20001108: 6,895,972 valid pixels } \\
\hline $\mathrm{BC}$ & 5.6 & 4.33 & 16.13 & 19.56 & 25.46 & 12.14 & 0.637 & 0.610 \\
\hline GIHS & 5.6 & 3.80 & 17.10 & 18.99 & 18.27 & 14.74 & 0.758 & 0.847 \\
\hline GIHSA & 5.3 & 3.41 & 18.18 & 21.47 & 21.27 & 14.78 & 0.778 & 0.823 \\
\hline MSW3 & 5.9 & 3.76 & 17.14 & 19.26 & 17.82 & 15.03 & 0.718 & 0.829 \\
\hline GS & 4.7 & 3.22 & 17.92 & 21.74 & 26.43 & 15.20 & 0.824 & 0.830 \\
\hline GSA & 4.6 & 3.18 & 17.86 & 21.73 & 26.33 & 15.42 & 0.833 & 0.846 \\
\hline MBTV & 5.8 & 3.93 & 16.71 & 20.18 & 21.21 & 13.55 & 0.741 & 0.759 \\
\hline Prop & 3.8 & 2.94 & 18.12 & 22.24 & 27.06 & 16.36 & 0.852 & 0.878 \\
\hline Prop-PC & 5.2 & 3.97 & 16.74 & 20.45 & 26.37 & 12.93 & 0.720 & 0.690 \\
\hline Prop-AP & 6.0 & 6.04 & 15.62 & 19.63 & 26.55 & 15.29 & 0.835 & 0.867 \\
\hline Prop-CLS & 3.8 & 2.97 & 17.89 & 22.11 & 27.16 & 16.37 & 0.846 & 0.877 \\
\hline Prop-NR & 5.6 & 3.85 & 15.14 & 18.40 & 19.47 & 15.78 & 0.730 & 0.861 \\
\hline
\end{tabular}

differences can be studied by viewing in a $1: 1$ scaling with a suitable image viewer. Images in electronic PDF versions of the paper, may be subject to compression or post-processing in the publication process which may mask actual differences or introduce spurious variations. Each figures' caption identifies the corresponding TIFF file.

From the images in Fig. 3 the HR capabilities of the IKONOS panchromatic imager are apparent; the panchromatic images show significant spatial detail and a number of features such as roads, buildings, etc can be readily resolved. By comparing the pan-sharpened images against the low-resolution $\mathrm{BC}$ version, we see that all of the pan-sharpening methods considered here offer a significant improvement over the lowresolution captured MS image by incorporating detail from the panchromatic image. The multi-resolution pan-sharpened multispectral images obtained with the proposed method (Prop) exhibit improved spatial detail compared with the CS methods
(GS), (GIHSA) - an effect that is best seen by viewing the image in its entirety. By focusing on smaller regions within the pan-sharpened images obtained by the methods, we can see that the proposed method (Prop) also exhibits improvements over the MBTV method. While the MBTV method preserves edges in the images well, it over-smooths the non-edge regions giving the corresponding pan-sharpened image in Fig. 3 a posterized appearance in which the regions bounded by edges appear artificially uniform in appearance. We observe that the pan-sharpened image obtained with the MSW3 method appears sharper than the proposed method (Prop) but upon closer examination reveals spatio-chromatic artifacts around strong color edges. These artifacts are not seen in the images obtained with the proposed method. For the images in Fig. 3 these artifacts are most apparent around the colored rooftops of the buildings in the scene (see Fig. S.4 in the Supplementary Materials). The artifacts in the wavelet-based 


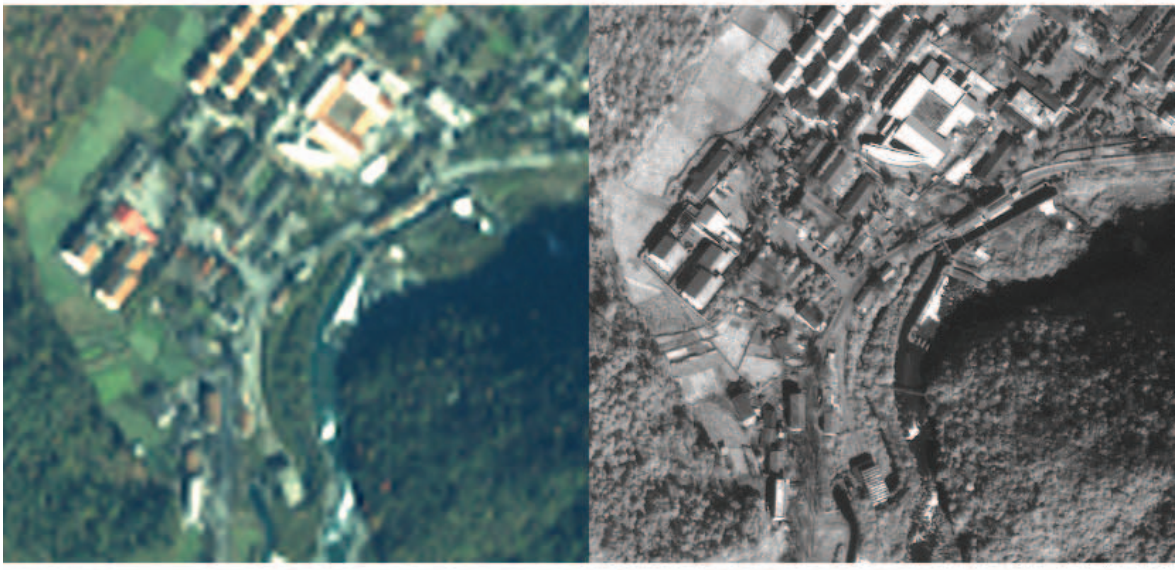

BC

PAN

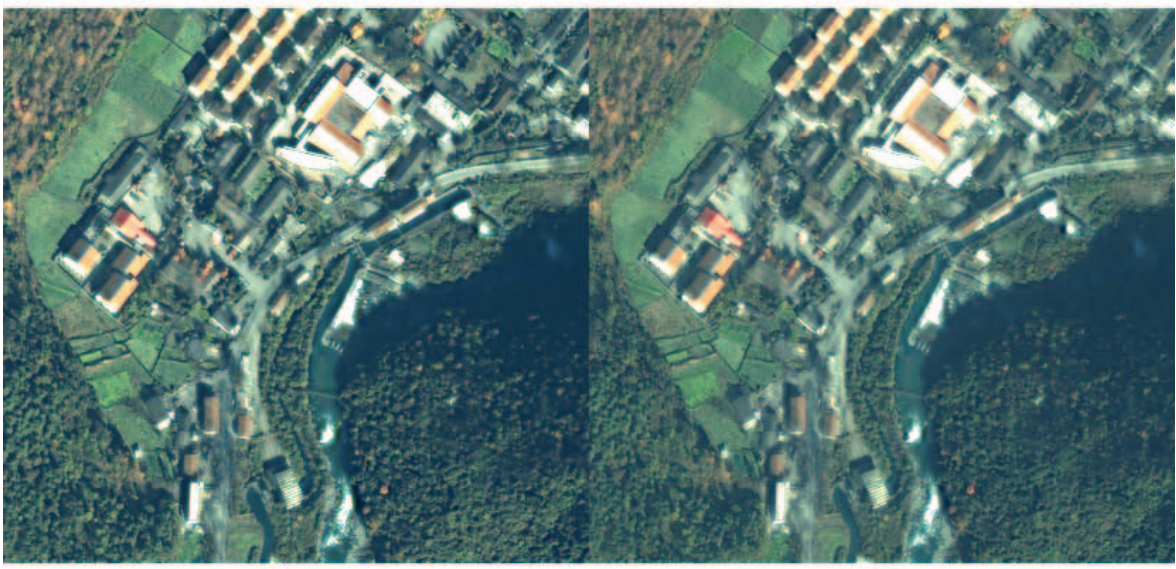

Prop

GS

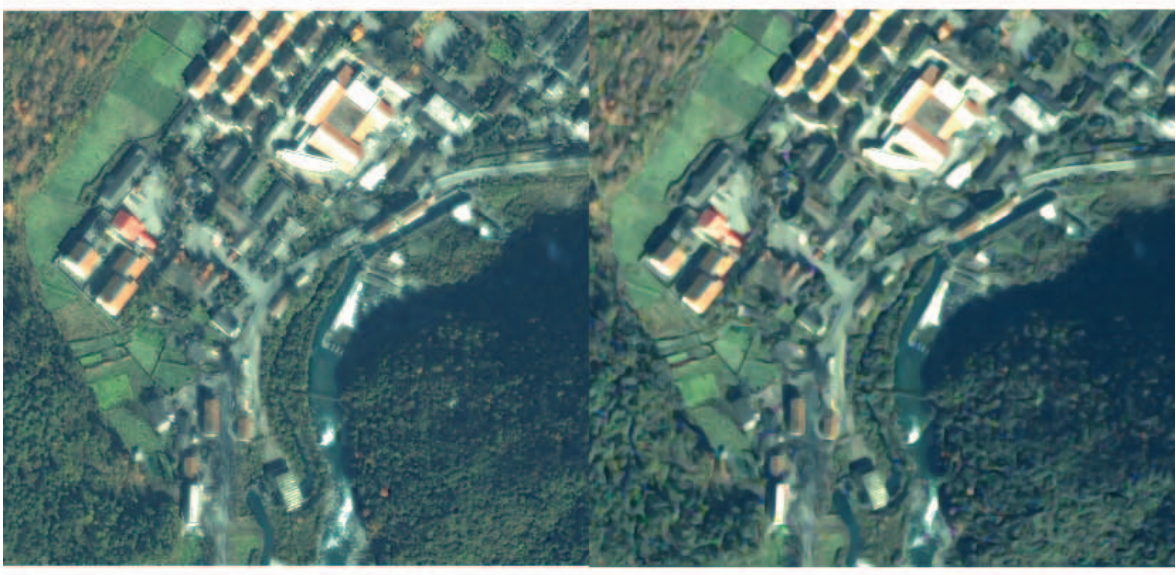

GIHSA

MBTV

Fig. 3. Sample pan-sharpening result for a portion of the China-Sichuan 58208_0000000.20001108 dataset (See FullResCompare1_58208_MS.tif) The R, G, and B spectral channels presented as a three channel image. Images are identified by the labels (placed below). See text for additional details. The corresponding NIR channel results can be found in the supplementary materials Fig. S.5 (File FullResCompare1_58208_NIR.tif).

pan-sharpening technique arise because the near-perfect reconstruction filterbanks [38] used in the wavelet based scheme are designed with matched forward and inverse transforms where aliasing introduced in the forward transform is canceled by the inverse transform. This alias cancellation property is, however, rendered ineffective in the wavelet pan-sharpening scheme by the substitution of the higher order bands in the spectral images from the panchromatic channel (whereas, the lower order subbands are retained from the spectral channel).
The spatio-spectral model in the proposed method, on the other hand, better represents the relation between the HR and the LR images.

\section{DISCUSSION}

The pan-sharpening method proposed in this paper is motivated and formulated based on explicit physically-motivated sensor models for the panchromatic and spectral channels. This is also the case for previously proposed techniques 
in the MBO framework but clearly distinct from methods in the CS and MRA frameworks where the sensor models are implicit rather than explicit. Compared with previously proposed techniques in the MBO framework [14]-[16], [19] the proposed formulation also presents several novelties and key differences, that we outline next.

- Variational pan-sharpening formulations are proposed in [14] and [19] that also use an objective function that is the sum of three parts, where the first term in (8) forms one of the three parts. The second term in (8) differs from its corresponding term in [14] and [19] in that the proposed term in (8) involves the spatial filter $\mathbf{G}_{0}$ and therefore impacts only the higher spatial frequency components, wheres the corresponding terms in [14] and [19] do not include a spatial filter and therefore impact all spatial frequency components. As already noted, the spatial filtering via $\mathbf{G}_{0}$ is desirable because it limits the impact of the second term to higher spatial frequencies and removes the influence of this term on low spatial frequencies where the observed multi-spectral images provide a better model than can be obtained by modeling the pan-chromatic channel as a linear combination of the spectral channels. Also, instead of the correlation based regularization term in (8), [14] uses an alternative regularization that is motivated by the underlying morphological assumption that the geometry, i.e., the edges, of the spectral channels are contained within the panchromatic image. The approach in [19] uses a total variation regularization term that encourages images that are piece-wise smooth between edges. Compared with these methods the proposed correlation-based regularization has the advantageous characteristic that the significance of the regularization term varies both based on spatial frequency because of the spatial filters $\mathbf{G}_{i}$, $i=1,2,3,4$ and based on the correlation of a given channel with the panchromatic image. The regularization reduces to a constrained least squares regularizer when the spectral channel is uncorrelated with the pan (See Section IV).

- An alternative MBO pan-sharpening approach is proposed in [15], by defining a Markov random field (MRF) inspired energy functional that is minimized subject to the constraints of the simple observation model in which pixels in captured LR images are assumed to be spatial averages of corresponding $q \times q$ pixel regions in the HR image. The spatial neighborhood weights for the MRF are computed from the panchromatic image to transfer edge information from the panchromatic image to the pansharpened images. Compared with [15], the formulation we propose in this paper uses a more refined and broadly accepted spatial model for the captured LR spectral images. The spatial regularization via the third term in (8) in our formulation inherently avoids conflict with the first observation model term whereas this is required as an explicit constraint in [15]. Also, [15] has no equivalent of the second term in (8).

- Another MBO pan-sharpening approach is proposed in [16], where the problem is formulated as a regularized constrained least squares restoration. Interpolated versions of the captured LR spectral images are treated as observed data and the standard linear spatially-invariant blur plus additive noise model used in image restoration is used to represent these images as degraded versions of the true HR spectral images. The observed panchromatic image is also modeled as a linear combination of the HR spectral images, after removal of the mean from all images. The restoration of the HR panchromatic image is then posed as the minimization of an objective function that additively combines the squared-errors in these two observation models with a constrained least squares regularization term. A discrete-sine transform (DST) is used to diagonalize the resulting system of equations and to obtain a closed form solution which is made computationally feasible using a block-based implementation. The method proposed in this paper, differs from [16] in several aspects. The observation model for the LR spectral channels explicitly incorporates downsampling (see next point, for additional elaboration). The second term in (8) corresponding to the square error for the observation model of the panchromatic image incorporates the high-pass spatial filter $\mathbf{G}_{0}$ and thereby avoids competition at low-spatial frequencies with the more accurate observation model for the spectral channels. The correlation-weighted regularization represented in the third term also serves to bring in spatial detail from the panchromatic channel instead of the constrained least squares regularizer that serves purely as a smoothing prior.

- The proposed approach also differs from the prior model based formulations in how the filters $\mathbf{H}_{i}, i=1,2, \ldots, K$ are determined. Specifically, these are non-square matrices estimated using the methodology described in [25] based on knowledge of the analog PSF for the desired HR and the captured LR spectral images and the spatial down-sampling factor $q$. The filters $\mathbf{H}_{i}$, $i=1,2, \ldots, K$ model potential aliasing in the process of mapping $f_{i}[\mathbf{x}], \quad \mathbf{x} \in \Lambda$ to $c_{i}[\mathbf{x}], \quad \mathbf{x} \in \Gamma$ in the observation model, which, in turn, allows for the aliasing to be partly resolved via the high frequency information contributed by the panchromatic image instead of being treated as noise in the data fidelity term. The utility of this approach has been demonstrated for the closely related image upsampling problem in [28]. In particular, this advantage cannot be realized with approaches that treat the spectral images interpolated up to the panchromatic resolution as the observed data [16] or use a simplistic model of the spatial relation between the images [15].

A comparison of the results for the proposed method (Prop) against those for the CS and MRA methods and for the other variants in our framework provides insight into the contributions of the different components in our formulation. The GS, GSA, and GIHSA methods that represent the state of the art in CS-based pan-sharpening methods, improve upon prior CS methods by better accounting for correlations between the spectral channels and the panchromatic channel. The proposed technique also accounts for the correlations 
between the spectral channels via the joint second term in (8) but improves upon the CS techniques, because of the more comprehensive spatial model in addition to the spectral model. Compared with the MSW3 MRA technique, the proposed method offers an improvement because the latter uses only an implicit spatial model and a rather simple spectral correlation model. The fact that the variants Prop-PC, Prop-AP, Prop-NR, and Prop-CLS do not perform as well as the complete proposed method (Prop) highlights the fact that each of the ingredients in the proposed method provides a benefit. The relative performance of the methods indicates, in particular, that the regularization term and the high pass filter for the joint second term in (8) both make a significant contribution to the improved performance of the proposed method. Introduction of correlation dependence in the regularization, provides a small benefit.

Methods in the CS and MRA frameworks have an advantage of much lower computational complexity compared with methods in the MBO framework in general and with the proposed method in particular. However, the much higher computational complexity of the proposed method is less of a concern for common situations where the pan-sharpened images are generated once and utilized many times over. In these settings, improving the quality of the pan-sharpened images is the predominant consideration over-riding the computational cost. Furthermore, parallel processing could be utilized to significantly reduce computation times. Although, the development of a parallel algorithm is beyond the scope of the present paper, we particularly note that the spatial filtering and re-sampling operations used in our iterations are inherent in common image processing tasks for which significant acceleration has been demonstrated using parallel processing, particularly, graphics processing units (GPUs).

\section{CONCLUSION}

A new model-based optimization approach is proposed that jointly determines pan-sharpened HR spectral images to minimize an objective function that combines squared residual error in physically motivated observation models of the LR multispectral and the HR panchromatic images and a regularization term. A computationally tractable iterative algorithm is introduced for solving the resulting optimization. The method is benchmarked against the commonly employed prior pan-sharpening methods. Numerical performance metrics and visual comparison validate that the method offers improved quality of pan-sharpened images, although at the cost of significant computational cost. A MATLAB ${ }^{\text {TMimplementation }}$ of the proposed pan-sharpening method is provided. ${ }^{9}$

\section{ACKNOWLEDGMENT}

The authors would like to thank the Associate Editor and the Reviewers for suggestions that have significantly improved the presentation and the Center for Integrated Research Computing, University of Rochester, for making available computational resources required for obtaining the results presented in this paper.

\footnotetext{
${ }^{9}$ The software is available at http://www.ece.rochester.edu/projects/siplab/ imaging.html
}

\section{REFERENCES}

[1] T. Ranchin, B. Aiazzi, L. Alparone, S. Baronti, and L. Wald, "Image fusion-The ARSIS concept and some successful implementation schemes," ISPRS J. Photogramm. Eng. Remote Sens., vol. 58, nos. 1-2, pp. 4-18, 2003.

[2] T. Ranchin and L. Wald, "Fusion of high spatial and spectral resolution images: The ARSIS concept and its implementation," Photogramm. Eng. Remote Sens., vol. 66, no. 1, pp. 49-61, 2000.

[3] C. Thomas, T. Ranchin, L. Wald, and J. Chanussot, "Synthesis of multispectral images to high spatial resolution: A critical review of fusion methods based on remote sensing physics," IEEE Trans. Geosci. Remote Sens., vol. 46, no. 5, pp. 1301-1312, May 2008.

[4] Y. Zhang, "Methods for image fusion quality assessment-A review, comparison and analysis," Int. Archives Photogramm., Remote Sens. Spatial Inf. Sci., vol. 37, Part B7, pp. 1101-1109, 2008.

[5] Y. Zhang, "Understanding image fusion," Photogramm. Eng. Remote Sens., vol. 70, no. 6, pp. 657-661, Jun. 2004.

[6] L. Alparone, L. Wald, J. Chanussot, C. Thomas, P. Gamba, and L. Bruce, "Comparison of pansharpening algorithms: Outcome of the 2006 GS-S data-fusion contest," IEEE Trans. Geosci. Remote Sens., vol. 46, no. 5, pp. 1301-1312, May 2008.

[7] Z. Wang, D. Ziou, C. Armenakis, D. Li, and Q. Li, "A comparative analysis of image fusion methods," IEEE Trans. Geosci. Remote Sens., vol. 43, no. 6, pp. 1391-1402, Jun. 2005.

[8] K. Nikolakopoulos, "Comparison of four different fusion techniques for IKONOS data," in Proc. IEEE Int. Symp. Geosci. Remote Sens., vol. 4. Sep. 2004, pp. 2534-2537.

[9] I. Amro, J. Mateos, M. Vega, R. Molina, and A. Katsaggelos, "A survey of classical methods and new trends in pansharpening of multispectral images," EURASIP J. Adv. Signal Process., vol. 79, pp. 1-22, Sep. 2011.

[10] C. Laben and B. Brower, "Process for enhancing the spatial resolution of multispectral imagery using pan-sharpening," U.S. Patent 6011875, Jan. 4, 2000.

[11] T. Tu, S. Su, H. Shyu, and P. Huang, "A new look at IHS-like image fusion methods," Inf. Fusion, vol. 2, no. 3, pp. 177-186, 2001.

[12] B. Aiazzi, S. Baronti, and M. Selva, "Improving component substitution pansharpening through multivariate regression of MS +Pan data," IEEE Trans. Geosci. Remote Sens., vol. 45, no. 10, pp. 3230-3239, Oct. 2007.

[13] K. Amolins, Y. Zhang, and P. Dare, "Wavelet based image fusion techniques-An introduction, review and comparison," ISPRS J. Photogramm. Remote Sens., vol. 62, no. 4, pp. 249-263, 2007.

[14] C. Ballester, V. Caselles, L. Igual, J. Verdera, and B. Rougé, "A variational model for P+XS image fusion," Int. J. Comput. Vis., vol. 69, no. 1 , pp. 43-58, 2006.

[15] H. Aanæs, J. Sveinsson, A. Nielsen, T. Bøvith, and J. Benediktsson, "Model-based satellite image fusion," IEEE Trans. Geosci. Remote Sens., vol. 46, no. 5, pp. 1336-1346, May 2008.

[16] Z. Li and H. Leung, "Fusion of multispectral and panchromatic images using a restoration-based method," IEEE Trans. Geosci. Remote Sens., vol. 47, no. 5, pp. 1482-1491, May 2009.

[17] L. Zhang, H. Shen, W. Gong, and H. Zhang, "Adjustable modelbased fusion method for multispectral and panchromatic images," IEEE Trans. Syst., Man, Cybern. B, Cybern., vol. 42, no. 6, pp. 1693-1704, Dec. 2012.

[18] F. Fang, F. Li, C. Shen, and G. Zhang, "A variational approach for pansharpening," IEEE Trans. Image Process., vol. 22, no. 7, pp. 2822-2834, Jul. 2013.

[19] F. Palsson, J. Sveinsson, and M. Ulfarsson, "A new pansharpening algorithm based on total variation," IEEE Geosci. Remote Sens. Lett., vol. 11, no. 1, pp. 318-322, Jan. 2014.

[20] H. Aly and G. Sharma, "Joint multichannel pansharpening for multispectral imagery," in Proc. IEEE ICASSP, Vancouver, BC, Canada, May 2013, pp. 2159-2163.

[21] M. Cook et al., "IKONOS technical performance assessment," in Algorithms for Multispectral, Hyperspectral, and Ultraspectral Imagery VII, vol. 4381, S. S. Shen and M. R. Descour, Eds. Bellingham, WA, USA: SPIE, Aug. 2001, pp. 94-108.

[22] S. Park, M. Park, and M. Kang, "Super-resolution image reconstruction: A technical overview," IEEE Signal Process. Mag., vol. 20, no. 3, pp. 21-36, May 2003.

[23] R. C. Gonzalez and P. Wintz, Digital Image Processing, 2nd ed. Reading, MA, USA: Addison-Wesley, 1987.

[24] E. Dubois, "The sampling and reconstruction of time-varying imagery with application in video systems," Proc. IEEE, vol. 73, no. 4, pp. 502-522, Apr. 1985. 
[25] H. A. Aly and E. Dubois, "Specification of the observation model for regularized image up-sampling," IEEE Trans. Image Process., vol. 14, no. 5 , pp. 567-576, May 2005.

[26] S. Geisser, Predictive Inference: An Introduction, vol. 55. Boca Raton, FL, USA: CRC Press, 1993.

[27] D. G. Luenberger, Linear and Nonlinear Programming, 2nd ed. Reading, MA, USA: Addison-Wesley, 1989.

[28] H. Aly and E. Dubois, "Image up-sampling using total-variation regularization with a new observation model," IEEE Trans. Image Process., vol. 14 , no. 10 , pp. $1647-1659$, Oct. 2005.

[29] G. Dial, H. Bowen, F. Gerlach, J. Grodecki, and R. Oleszczuk, "IKONOS satellite, imagery, and products," Remote Sens. Environ., vol. 88, no. 1, pp. 23-36, 2003.

[30] Space-Imaging, Thornton, CO, USA. (2000). Geoeye IKONOS Scene Data [Online]. Available: http://ftp://ftp.glcf.umiacs.umd.edu/glcf

[31] A. Garzelli, F. Nencini, L. Alparone, B. Aiazzi, and S. Baronti, "Pan-sharpening of multispectral images: A critical review and comparison," in Proc. IEEE Int. Symp. Geosci. Remote Sens., vol. 1. Sep. 2004 pp. $81-84$.

[32] L. Wald, "Quality of high resolution synthesised images: Is there a simple criterion?" in Proc. 3rd Conf. Fusion Earth Data, Jan. 2000, pp. $99-103$.

[33] A. Bovik, Handbook of Image and Video Processing. New York, NY, USA: Academic, 2000

[34] L. Wald, T. Ranchin, and M. Mangolini, "Fusion of satellite images of different spatial resolutions: Assessing the quality of resulting images," Photogramm. Eng. Remote Sens., vol. 63, no. 6, pp. 691-699, 1997.

[35] L. Alparone, B. Aiazzi, S. Baronti, A. Garzelli, F. Nencini, and M. Selva, "Multispectral and panchromatic data fusion assessment without reference," Photogramm. Eng. Remote Sens., vol. 74, no. 2, p. 193, 2008.

[36] Z. Wang and A. Bovik, "A universal image quality index," IEEE Signal Process. Lett., vol. 9, no. 3, pp. 81-84, Mar. 2002.

[37] L. Alparone, S. Baronti, A. Garzelli, and F. Nencini, "A global quality measurement of pan-sharpened multispectral imagery," IEEE Geosci. Remote Sens. Lett., vol. 1, no. 4, pp. 313-317, Oct. 2004

[38] P. P. Vaidyanathan, Multirate Systems and Filter Banks. Englewood Cliffs, NJ, USA: Prentice-Hall, 1993.

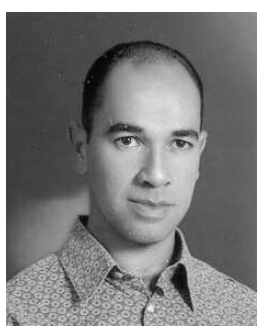

Hussein A. Aly (S'11) received the B.Sc. (Hons.) degree in computer engineering and the M.Sc. degree in electrical engineering from the Military Technical College (MTC), Egypt, and the Ph.D. degree in electrical engineering from the University of Ottawa in 1993, 1997, and 2004, respectively. $\mathrm{He}$ is an Associate Professor with the Computer Department at MTC, and was the Department Chief from 2010 to 2013. He was a Visiting Professor with the Department of Electrical and Computer Engineering, University of Rochester, from 2012 to 2013. His research interests are in image sampling theory and sampling structure conversion. His current research is focused on high-quality image magnification, interpolation of color filter array data, the application of totalvariation for image processing, data fusion, and video steganography. While at the University of Ottawa, he participated in a project on image magnification funded by the Royal Canadian Mounted Police. He is a Senior Member of the IEEE Signal Processing Society and the Egypt Engineers Syndicate.

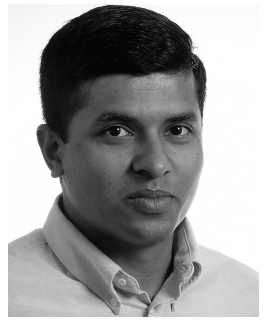

Gaurav Sharma (''88-M'96-SM'00-F'13) is an Associate Professor with the Department of Electrical and Computer Engineering, the Department of Biostatistics and Computational Biology, and the Department of Oncology, University of Rochester. From 2008 to 2010, he served as the Director of the Center for Emerging and Innovative Sciences, a New York state funded center for promoting joint university-industry research and technology development, which is housed at the University of Rochester. $\mathrm{He}$ received the B.E. degree in electronics and communication engineering from IIT Roorkee (formerly University of Roorkee), India, the M.E. degree in electrical communication engineering from the Indian Institute of Science, Bangalore, India, and the M.S. degree in applied mathematics and the Ph.D. degree in electrical and computer engineering from North Carolina State University, Raleigh, in 1990, 1992, 1995, and 1996, respectively. From 1996 to 2003, he was with Xerox Research and Technology, Webster, NY, USA, as a member of the research staff and a Principal Scientist.

Dr. Sharma's research interests include image processing, media security, distributed signal processing, and bioinformatics. He is the Editor of the Color Imaging Handbook (CRC press, 2003). He is a fellow of SPIE and the Society of Imaging Science and Technology (IS\&T), and a member of Sigma Xi, Phi Kappa Phi, Pi Mu Epsilon, and the signal processing and communications societies of the IEEE. He served as a Technical Program Chair of the IEEE International Conference on Image Processing in 2012, the Symposium Chair of the SPIE/IS\&T Electronic Imaging Symposium in 2013, the Chair of the IEEE Signal Processing Society's Image, Video, and Multidimensional Signal Processing Technical Committee from 2010 to 2011, the Chair of the Rochester Section of the IEEE in 2007, and the Chair of the Rochester Chapter of the IEEE Signal Processing Society in 2003. He is the Editor-inChief of the Journal of Electronic Imaging, and served as an Associate Editor of the Journal of Electronic Imaging, the IEEE TRANS ACTIONS ON IMAGE PROCESSING, and the IEEE TRANSACTIONS ON INFORMATION FORENSICS AND SECURITY. 